\title{
Solving Two Problems IN Number Theory
}

\author{
Mykhaylo Khusid
}

\begin{abstract}
In 1995, Olivier Ramaret proved that any even number is the sum of no more than 6 primes. From the validity of Goldbach's ternary hypothesis (proved in 2013 year) it follows that any even number is the sum of not more than 4 numbers [1]. In the article, the author confirms the above and proves that the cause and effect of this is any even number the sum of not more than two prime and twin primes are infinite [8]-[14].
\end{abstract}

Index Terms - solving two problems in number theory.

\section{THEOREM 1}

Any even number starting from 12 is representable as a sum four odd prime numbers.

1. For the first even number $12=3+3+3+3$. We allow justice for the previous $\mathrm{N}>5$ :

$$
p_{1}+p_{2}+p_{3}+p_{4}=2 N
$$

We will add to both parts on 1

$$
p_{1}+p_{2}+p_{3}+p_{4}+1=2 N+1
$$

where on the right the odd number also agrees:

$$
p_{1}+p_{2}+p_{3}+p_{4}+1=p_{5}+p_{6}+p_{7}
$$

Having added to both parts still on 1:

$$
p_{1}+p_{2}+p_{3}+p_{4}+2=p_{5}+p_{6}+p_{7}+1
$$

We will unite $p_{6}+p_{7}+1$. Again we have some odd number, which according to (1)we replace with the sum of three simple and as a result we receive:

$$
p_{1}+p_{2}+p_{3}+p_{4}+2=p_{5}+p_{6}+p_{7}+p_{8}
$$

At the left the following even number is relative (1), and on the right the sumfour prime numbers.

$$
p_{1}+p_{2}+p_{3}+p_{4}=2 N
$$

Thus, obvious performance of an inductive mathematical method. As was to be shown. And the sum of all sorts of odd number we have any even number, starting from 18 :

$$
p_{1}+p_{2}+p_{3}+p_{4}+p_{5}+p_{6}=2 N
$$

The proof of the binary Goldbach problem proposed earlier by the author is not true (Resources used: [2]-[6]). The author apologizes and represents correct solution.

\section{THEOREM 2 (STRAIGHT)}

Representing any even number as the sum of 6 and 4 odd primes is a solution to the strong Goldbach problem. From (6), (7) it follows:

$$
\begin{aligned}
& p_{1}+p_{2}+p_{3}+p_{4}+p_{5}+p_{6}=p_{7}+p_{8}+p_{9}+p_{10} \\
& p_{1}+p_{2}+p_{3}+p_{4}+p_{5}+p_{6}=2 N
\end{aligned}
$$

where $2 \mathrm{~N} \geqslant 18, \mathrm{~N} \geqslant 9$.

$$
p_{11}+p_{12}+p_{13}+p_{14}=2 N_{1}
$$

where $2 \mathrm{~N}_{1} \geq 12, \mathrm{~N}_{1} \geq 6$.

Since $N$ and $N_{l}$ have all possible values, then:

$$
2 N-2 N_{1}=p_{7}+p_{8}, p_{7}+p_{8} \leq p_{9}+p_{10}
$$

which means the sum of six odd primes is equivalent to sum, where two primes from the sum of four and:

$$
p_{11}+p_{12}+p_{13}+p_{14}=p_{9}+p_{10}
$$

Thus, from (12) it follows that the sum of four primes is equal to the sum of two simple.

\section{THEOREM 3. (CONVERSE)}

Goldbach's binary hypothesis is correct. Otherwise, the sum of six odd prime numbers or, and the sum of four cannot be represented by any even number.

Let be :

$$
p_{11}+p_{12}+p_{13}+p_{14} \neq p_{9}+p_{10}
$$

And since the sum of two odd prime numbers is an even number, equal to Since it is impossible to deny any pair of any two even numbers (11), then it turns out that the sum of six arbitrary odd primes is not equal to the sum of four arbitrary odd primes (8), which is also impossible. From what follows the inevitability of the equality of the sum of four primes to the sum of two primes. Thus, the equality of the sum of six primes and four is equivalent to the equality of the 
sum of four odd primes two and is equal to $2 \mathrm{~N}$, where $2 N \geq 18$ and the equality of the sum of 6 and 4 primes to any even number is a consequence of the equality to any even number of the sum of two primes and vice versa.

An even number greater and equal to 18 , which cannot be represented as the sum of two simple odd numbers do not exist Representation of even numbers from 6 to 18 (minimum sum of 6 odd prime numbers) we show arithmetically with two odd prime numbers. Any even number starting with six is the sum of two prime numbers. The Goldbach-Euler conjecture is correct and proven.

Thus, we proved:

Any even number since 6 is representable in the form of a bag of two odd the simple.

$$
p_{1}+p_{2}=2 N
$$

Any even number is representable in the form of the sum of two simple. In total even numbers, without exception, since 6 are the sum of two prime numbers.

Goldbakha-Euler's problem is true and proved!

Theorem the four simple and the Goldbach Euler conjecture have a series of corollary. One of which is relevant problem in number theory.

Corollary. If the sum of two primes from the sum of four $2 \mathrm{~N}$, starting from 12 , set in the open interval [6,2N-6], then the sum the remaining two simple variables are the required even number. What can be seen from the proven GoldbachEuler hypothesis.

\section{The prime numbers of twins are infinite.}

Any even number, starting from 14, can be represented as a sum four odd primes, of which two primes are twins.

$$
p_{1}+p_{2}+p_{3}+p_{4}=2 N
$$

Let $p_{3}, p_{4}$ prime numbers be twins, then the difference is any even, starting at 14 , and the sum of the primes of the twins is also an even number, which, according to the proved Goldbach-Euler hypothesis, is equal to the sum of two prime numbers (Corollary).

Next, we place the prime numbers from left to right in descending order. And if the even number
$2 N=2 p_{2}+2 p_{4}+4$, then $p_{1}, p_{2}$ inevitably also twins. Subtract the sum from both parts (15) $2 p_{2}+2 p_{4}$ :

$$
p_{1}-p_{2}+p_{3}-p_{4}=4
$$

From (16), it is obvious - inevitably twins. Let their finite number and the last prime numbers be twins $p_{3}, p_{4}$. Denote two primes greater than $p_{3}, p_{4}$ how $p_{1}, p_{2}$. Sum up all four primes and then according to the sum theorem four simple there is an even number $2 \mathrm{~N}$ at which inevitably large $p_{1}, p_{2}$ -twins. And then substituting $p_{3}, p_{4}$ numeric values instead $p_{1}, p_{2}$ in (15) the process becomes infinite and the prime numbers are twins - infinite number.

\section{REFERENCES}

[1] https://ru.wikipedia.org/wiki/\%D0\%9F\%D1\%80\%D0\%BE\%D0\%B1 $\% \mathrm{D} 0 \% \mathrm{BB} \% \mathrm{D} 0 \% \mathrm{~B} 5 \% \mathrm{D} 0 \% \mathrm{BC} \% \mathrm{D} 0 \% \mathrm{~B} 0 \_\% \mathrm{D} 0 \% 93 \% \mathrm{D} 0 \% \mathrm{BE} \% \mathrm{D} 0 \%$ $\mathrm{BB} \% \mathrm{D} 1 \% 8 \mathrm{C} \% \mathrm{D} 0 \% \mathrm{~B} 4 \% \mathrm{D} 0 \% \mathrm{~B} 1 \% \mathrm{D} 0 \% \mathrm{~B} 0 \% \mathrm{D} 1 \% 85 \% \mathrm{D} 0 \% \mathrm{~B} 0$

[2] http://molodyvcheny.in.ua/files/conf/other/33feb2019/67.pdf.

[3] http://www.ijma.info/index.php/ijma/article/view/5973.

[4] https://www.ijma.info/index.php/ijma/article/view/6048/3565.

[5] https://doi.org/10.30525/978-9934-588-11-2_17.

[6] https://ppublishing.org/upload/iblock/977/EJT-6_2018.pdf, page 1819.

[7] http://molodyvcheny.in.ua/files/conf/other/49july2020/20.pdf.

[8] Chen, J.R. "On the Distribution of Almost Primes in an Interval."Sci Sinica18, 611-627, 1975.

[9] Euler, L. "De numeris primis valde magnis."Novi Commentarii academiae scientiarum Petropolitanae9, 99-153, (1760) 1764 Reprinted in Commentat. arithm.1, 356-378, 1849. Reprinted in Opera Omnia: Series 1, Volume 3, pp.1-45.

[10] Goldman, J.R.The Queen of Mathematics: An Historically Motivated Guide to Number Theory.Wellesley, MA: A K Peters, p. 22, 1998.

[11] Hardy, G.H. and Wright, W.M. "Unsolved Problems Concerning Primes." $\$ 2.8$ and Appendix $\$ 3$ in An Introduction to the Theory of Numbers, 5th ed.Oxford, England: Oxford University Press, pp. 19 and 415-416, 1979.

[12] Iwaniec, H. and Pintz, J. "Primes in Short Intervals."Monatsh. f. Math.98, 115-143, 1984.

[13] Sloane, N.J.A. Sequences A002496/M1506, A005574/M1010, and A007491/M1389 in "The On-Line Encyclopedia of Integer Sequences".

[14] Lamb E. Goldbach Variations [Text]/ E.Lamb Scientific American blogs - May 15, 2013. 